\title{
Valorización de residuos de la obtención de almidón de ñame espino para su uso como bioadsorbente en la remoción de Cromo (VI) y Níquel (II)
}

\section{Waste valorization of starch obtained from hawthorn yam as bioadsorbent on Chromium (VI) and Nickel (II) removal}

DOI: http://doi.org/10.17981/ingecuc.16.1.2020.02

Artículo de Investigación Científica. Fecha de Recepción: 13/06/2019. Fecha de Aceptación: 27/01/2020

\author{
Angel Villabona-Ortíz (i) \\ Universidad de Cartagena. Cartagena (Colombia) \\ avillabonao@unicartagenaedu.co \\ Candelaria Tejada-Tovar \\ Universidad de Cartagena. Cartagena (Colombia) \\ ctejadat@unicartagena.edu.co \\ Paula Ramírez-Vasquez (1) \\ Universidad de Cartagena. Cartagena (Colombia) \\ paularava04@hotmail.com
}

Para citar este artículo:

A. Villabona Ortíz, C. Tejada-Tovar y P. Ramírez-Vasquez, "Valorización de residuos de la obtención de almidón de ñame espino para su uso como bioadsorbente en la remoción de Cromo (VI) y Níquel (II)”, INGE CUC, vol. 16, no. 1, pp. 22-33, 2020. DOI: http://doi.org/10.17981/ingecuc.16.1.2020.02

\section{Resumen}

Introducción- Entre los metales pesados descargados en fuentes hídricas se encuentran el Cromo (VI) y el Níquel (II), los cuales causan efectos peligrosos a la salud.

Objetivo- Optimizar el efecto de la concentración inicial de contaminante, temperatura y dosis de adsorbente usando residuos del proceso de obtención de almidón de ñame espino (D. rotundata) en la remoción de Cromo (VI) y Níquel (II).

Metodología- Se optimizó aplicando la Metodología Superficie Respuesta (RSM), realizando el estudio cinético y de equilibrio a la condición óptima encontrada, evaluando el ajuste de los datos de cinética a los modelos de pseudo-primer orden, pseudo-segundo orden, Elovich; los de isotermas a los modelos de Langmuir y Freundlich. Se calcularon los parámetros termodinámicos- Energía libre de Gibbs $\left(\Delta \mathrm{G}^{\circ}\right)$, Entalpía $\left(\Delta \mathrm{H}^{\circ}\right)$ y Entropía $\left(\Delta \mathrm{S}^{\circ}\right)$, por el método gráfico de Van't Hoff.

Resultados- De la MSR se encontró que las condiciones óptimas para $\mathrm{Cr}(\mathrm{VI})$ fueron $76.6{ }^{\circ} \mathrm{C}, 0.14 \mathrm{~g}$ y $368,18 \mathrm{ppm}$, y para $\mathrm{Ni}(\mathrm{II}) 70^{\circ} \mathrm{C}, 1,19$ g y $31,82 \mathrm{ppm}$. La capacidad de adsorción máxima fue de $66,25 \mathrm{mg} / \mathrm{g}$ de $\mathrm{Cr}(\mathrm{VI})$ y $17.67 \mathrm{mg} / \mathrm{g}$ de $\mathrm{Ni}(\mathrm{II})$ El modelo cinético de pseudo-segundo orden ajusta los datos de adsorción de $\mathrm{Cr}(\mathrm{VI})$ y el de Elovich ajusta los de $\mathrm{Ni}(\mathrm{II})$; por su parte el modelo de isoterma de Freundlich mostró el mejor ajuste de los datos de adsorción de los iones en estudio. De los valores de $\Delta \mathrm{G}^{\circ}, \Delta \mathrm{S}^{\circ}$, y $\Delta \mathrm{H}^{\circ}$ se establece que el proceso para $\mathrm{Cr}(\mathrm{VI})$ es endotérmico, no espontáneo no favorable y reversible; para Ni(II) que es no espontaneo, exotérmico y controlado por fisisorción.

Conclusiones- Los residuos del proceso de extracción de almidón de ñame son un adsorbente efectivo para la remoción de $\mathrm{Cr}(\mathrm{VI})$ y $\mathrm{Ni}(\mathrm{II})$ presentes en solución acuosa y la MSR arrojó valores óptimos que sirven de base para la escalabilidad del proceso.

Palabras clave- Cinética; Dioscorea rotundata; Isotermas de adsorción, Metodología superficie respuesta, Optimización

\section{Abstract}

Introduction- Among the heavy metals discharged on water sources the Chromium (VI) and Nickel (II) cause dangerous health effects.

Objective- Optimize the effect of the initial concentration of pollutant, temperature and adsorbent dose using residues of the starch obtaining process from hawthorn yam (D. rotundata) in the removal of Chromium (VI) and Nickel (II).

Method- The optimization was carried out by applying the Response Surface Methodology (RSM), performing the kinetic and equilibrium study to the optimal condition found, evaluating the fit of the kinetic data to the pseudo-first order, pseudo-second order and Elovich models; those of isotherms to the models of Langmuir and Freundlich. The thermodynamic parametersGibbs free Energy $\left(\Delta \mathrm{G}^{\circ}\right)$, Enthalpy $\left(\Delta \mathrm{H}^{\circ}\right)$ and Entropy $\left(\Delta \mathrm{S}^{\circ}\right)$, were calculated using the Van't Hoff graphical method.

Results- From the MSR it was found that the optimal conditions for $\mathrm{Cr}(\mathrm{VI})$ were $76.6^{\circ} \mathrm{C}, 0.14 \mathrm{~g}$ and $368.18 \mathrm{ppm}$, and for $\mathrm{Ni}$ (II) $70{ }^{\circ} \mathrm{C}, 1.19 \mathrm{~g}$ and $31.82 \mathrm{ppm}$. The maximum adsorption capacity was $66.25 \mathrm{mg} / \mathrm{g} \mathrm{Cr}(\mathrm{VI})$ and $17.67 \mathrm{mg} / \mathrm{g} \mathrm{Ni}(\mathrm{II})$. The pseudo-second order kinetic model adjusts the adsorption data of $\mathrm{Cr}(\mathrm{VI})$ and that of Elovich adjusts the Ni(II) data; on the other hand, the Freundlich isotherm model showed the best adjustment of the adsorption data of the ions under study. The values of $\Delta \mathrm{G}^{\circ}, \Delta \mathrm{S}^{\circ}$, and $\Delta \mathrm{H}^{\circ}$ established that the process for $\mathrm{Cr}(\mathrm{VI})$ is endothermic, not spontaneous, not favorable and reversible; for $\mathrm{Ni}$ (II) which is non-spontaneous, exothermic and controlled by physisorption.

Conclusions- The residues of the hawthorn yam starch extraction process are an effective adsorbent for the removal of $\mathrm{Cr}$ (VI) and Ni (II) present in aqueous solution and the MSR yielded optimal values that serve as the basis for the scalability of the process.

Keywords- Kinetics; Dioscorea rotundata; Adsorption isotherms, Surface response methodology, Optimization 


\section{INTRODUCCIÓN}

La no degradabilidad, la toxicidad y la bio-magnificación de los metales pesados los convirtieron en una amenaza tanto para los seres humanos como para los ecosistemas, ya que se presentan en bajas concentraciones en organismos al principio de la cadena trófica y en mayor proporción a medida que se asciende en la misma [1]. Las aguas residuales industriales contaminadas con metales pesados provenientes de industrias como el metalizado, las curtiembres, las operaciones mineras, las industrias de galvanoplastia, las industrias de fertilizantes, pesticidas y baterías, son los que más aportan a la presencia de estos contaminantes en fuentes hídricas [2].

Entre los metales descargados en fuentes hídricas se encuentran el Cromo (VI) y el Níquel (II), los cuales causan efectos peligrosos en nuestra salud. El Cromo (VI) es de naturaleza cancerígena y produce cáncer en los pulmones, la nariz y los senos nasales; la concentración máxima permitida de acuerdo a la US-EPA es de 0,05 mg/L [3], [4]. De otra parte, el Níquel (II) puede causar asma y enfermedades cutáneas; y en su mayoría, la contaminación del níquel es causada por minerales de níquel y sulfuro, también se encuentra en los compuestos de hierro, cobre, cobalto y algunos otros metales; y su nivel máximo permitido de acuerdo a la Organización Mundial de la Salud (OMS) es de 0,07 $\mathrm{mg} / \mathrm{g}[5]$.

Convencionalmente se han usado métodos fisicoquímicos como la precipitación química, intercambio iónico, electrocoagulación, ultra filtración, oxidación, fotocatálisis, entre otros; cuyo principal inconveniente es que resultan ser costosos y generan gran cantidad de lodos [6], [7]. Por esta razón, existe la necesidad de un método de tratamiento para la eliminación de metales en aguas residuales que sea simple, eficaz y de bajo costo. En éste sentido, los procesos de adsorción se convierten en opción, ya que es un método de tratamiento simple para eliminar iones metálicos en aguas residuales; sin embargo, el costo asociado con los adsorbentes comerciales hacen costoso el proceso de adsorción, lo que ha llevado a la búsqueda de nuevas estrategias para el desarrollo de materiales de bajo costo con una buena capacidad de remoción [8]. Como resultado de lo anterior se han usado residuos agrícolas y agroindustriales como adsorbentes naturales para la eliminación de metales pesados por su biodegradabilidad, sostenibilidad, bajo costo, capacidad de renovación y superficie rica con grupos funcionales [9]. Se han usado diferentes biomasas en la remoción de $\mathrm{Cr}$ (VI) y $\mathrm{Ni}$ (II), tales como chicharos [1], paja de arroz [10]-[11], limón, lanzón [12], cáscaras de litchi [13], cáscara de nuez de roble [14], entre otros.

Debido a la iniciativa del gobierno de Colombia de incentivar la siembra de ñame en zonas afectadas por el conflicto armado, con miras a la exportación y satisfacción del consumo nacional, se motivó la investigación en torno a la línea de producción de ñame espino postcosecha, que no cumple los estándares para consumo humano, y que formará parte de varios sub-procesos en una bio-refinería proyectada para el departamento de Bolívar, donde se busca aprovechar y optimizar los recursos, materias primas y residuos a partir de dicha materia prima [15]. Por esta razón, es importante contar con alternativas de aprovechamiento de los residuos del proceso en mención, donde el uso como biomaterial adsorbente es una buena opción, teniendo en cuenta que el rendimiento de la extracción del almidón es cercano al 20\% [16]. Es por esto que el objetivo del presente proyecto fue optimizar el efecto de la concentración inicial de contaminante, temperatura y dosis de adsorbente mediante la Metodología Superficie Respuesta (RSM), usando residuos de la obtención de almidón de ñame espino (D. rotundata) en la remoción de Cromo (VI) y Níquel (II). El mecanismo de adsorción se evaluó mediante el ajuste de los datos experimentales de cinética a los modelos de pseudo-primer orden, pseudo-segundo orden y Elovich. El equilibrio de adsorción se estudió ajustando los datos a los modelos de Langmuir y Freundlich. Y se calcularon los parámetros termodinámicos Energía libre de Gibbs $\left(\Delta \mathrm{G}^{\circ}\right)$, Entalpía $\left(\Delta \mathrm{H}^{\circ}\right)$ y Entropía $\left(\Delta S^{\circ}\right)$, para establecer la espontaneidad, favorabilidad y la influencia de la temperatura en el proceso. Éste estudio básico servirá de soporte para el futuro escalamiento del proceso en mención. 


\section{MetodoloGía}

\section{A. Preparación de la biomasa}

El ñame residual se recolectó en San Juan Nepomuceno-Bolívar (Colombia). Para la obtención de la pulpa residual se peló, lavó con agua destilada, luegose sumergió en una solución de $\mathrm{NaOH}$ al $0,25 \%$ a $5{ }^{\circ} \mathrm{C}$ durante 18 horas, y posteriormente se licuó durante 1 minuto, obteniendo un producto rico en almidón, al cual se le ajustó el pH y se reparó de la pulpa residual mediante filtración; éste material fue secado al sol durante 8 horas [17].

\section{B. Preparación de las soluciones sintéticas}

Se prepararon soluciones madres de $\mathrm{Cr}$ (VI) y Ni (II) a 1000 ppm cada una, usando como reactivos base dicromato de potasio $\left(\mathrm{K}_{2} \mathrm{Cr} 2 \mathrm{O} 7\right)$ y sulfato de Níquel (NiSO4). Luego, fueron diluidas para alcanzar la concentración de trabajo; para los ensayos experimentales se fijó pH 2 y 6 para $\mathrm{Cr}(\mathrm{VI})$ y $\mathrm{Ni}(\mathrm{II})$, respectivamente [18]-[20].

\section{Diseño experimental aplicado a la optimización de variables del proceso}

El procedimiento experimental, diseñado se formuló por medio del software Statgraphics Centurion XVI. II de factor continuo lineal, en superficie de respuesta de tipo compuesto central estrella, para determinar la mejor combinación de parámetros operacionales, que conllevó a un valor de respuesta óptima mediante la Metodología de Superficie de Respuesta (MSR), obteniendo así una ecuación de regresión que ajustó todos los datos del sistema. En la Tabla 1 , se muestran los rangos experimentales y niveles de variación de las variables. La respuesta a optimizar fue el porcentaje retenido de Cromo y Níquel (\%R) [19].

TABla 1. Diseño EXPERIMENTAL TIPO FACTORIAL.

\begin{tabular}{|l|l|l|l|l|l|}
\hline \multirow{2}{*}{\multicolumn{1}{|c|}{ Variables independientes }} & \multicolumn{5}{c|}{ Rango y nivel } \\
\cline { 2 - 7 } & \multicolumn{1}{|c|}{$-\mathrm{a}$} & \multicolumn{1}{|c|}{-1} & \multicolumn{1}{c|}{0} & \multicolumn{1}{c|}{+1} & \multicolumn{1}{c|}{$+\mathrm{\alpha}$} \\
\hline Temperatura $\left({ }^{\circ} \mathrm{C}\right)$ & 30 & 40 & 55 & 70 & 80 \\
\hline Cantidad de material adsorbente $(\mathrm{g})$ & 0,135 & 0,355 & 0,678 & 1 & 1,22 \\
\hline Concentración inicial $(\mathrm{ppm})$ & 31,82 & 100 & 200 & 300 & 368,18 \\
\hline
\end{tabular}

Fuente: Autores

\section{Cinética de adsorción}

Se colocó en contacto la biomasa en estudio con $100 \mathrm{~mL}$ de solución de $\mathrm{Cr}$ (VI) y Ni(II) a las condiciones óptimas encontradas luego de aplicar la MSR, con agitación a 200 rpm, y se tomaron muestras del metal en un rango entre 10 y 300 min para conocer el comportamiento de la cinética de eliminación de cada metal. La medición del $\mathrm{Cr}$ (VI) en solución después del proceso de adsorción se realizó mediante espectrometría UV-Vis a $540 \mathrm{~nm}$ usando Difenil Carbazida como reactivo de contraste [21]; y el Ni(II) se determinó por absorción atómica a $305.1 \mathrm{~nm}$. Los datos obtenidos se ajustaron a los modelos experimentales utilizando regresión no lineal en el software OriginPro, teniendo como criterio de ajuste el coeficiente de correlación $\mathrm{R}^{2}$. Los experimentos cinéticos indican la duración necesaria para alcanzar la condición de equilibrio, así los modelos de pseudo primer (1), pseudo segundo orden (2) y Elovich (3) fueron ajustados para analizar los datos experimentales de cinética.

\section{1) Modelo cinético de pseudo primer orden}

La expresión matemática desarrollada por Lagergren y correspondiente a la cinética de primer orden reversible, se basa en la suposición de que a cada ión metálico se le asigna un sitio de adsorción del material adsorbente, lo cual en términos de velocidad de reacción se expresa [6]:

$$
q_{t}=q_{e}\left(1-e^{-k_{1} t}\right)
$$


Donde $\mathrm{q}_{\mathrm{e}} \mathrm{y} \mathrm{q}_{\mathrm{t}}$ son las capacidades de adsorción en el equilibrio, $\mathrm{y}$ en un tiempo t, respectivamente expresadas en ( $\mathrm{mmol} / \mathrm{g})$, mientras $\mathrm{k} 1$ es la constante de pseudo primer orden $\left(\mathrm{min}^{-1}\right)$ [22].

2) Modelo cinético de pseudo segundo orden

Modelo desarrollado por Ho y McKay en 1999, en el que se supone que el adsorbato se adsorbe en dos sitios activos de la biomasa y el proceso es controlado por quimisorción; y la ecuación de velocidad de la cinética de adsorción se expresa [4]:

$$
q_{t}=\frac{t}{\frac{1}{k_{2} q_{e}^{2}}+\frac{t}{q_{e}}}
$$

Donde $\mathrm{k}_{2}$ es la constante de adsorción de segundo orden $\left(\mathrm{g}^{-1} \mathrm{~min}^{-1}\right)$, esta constante es obtenida de la gráfica de t/qt vs t [23].

3) Modelo de Elovich

Este modelo supone que los sitios activos del bioadsorbente son heterogéneos y por ello exhiben diferentes energías de activación, basándose en un mecanismo de reacción de segundo orden para un proceso de reacción heterogénea, y está descrito por la ecuación 3 [24]:

$$
q_{t}=\frac{1}{\beta} \ln (\alpha \beta)+\frac{1}{\beta} \ln (t)
$$

Donde $a$ es la velocidad de adsorción inicial del modelo de Elovich (mg/g min) y $\beta$ es la constante relacionada con el alcance de la cobertura superficial y la energía de activación en la quimisorción $(\mathrm{g} / \mathrm{mg})[25]$.

\section{E. Isotermas de adsorción}

En orden de entender la distribución de los iones metálicos en la fase liquida y sólida en el equilibrio fueron estudiadas las mismas condiciones de los estudios cinéticos al tiempo de equilibrio. Se hizo uso de los modelos de Langmuir (4) y Freundlich (5) para ajustar a los datos experimentales obtenidos al tiempo de equilibrio.

\section{1) Modelo de Langmuir}

El modelo de Langmuir supone que el metal es tomado en una superficie homogénea, es decir una adsorción en monocapa sin interacción entre las moléculas adsorbidas, así como, energías de adsorción uniformes en la superficie y la no migración del adsorbato hacia otros sitios [22], y se expresa de acuerdo a la ecuación (4):

$$
q_{e}=q \max \frac{b c_{e}}{1+b C_{e}}
$$

Donde $q_{\mathrm{e}}$ es la concentración del metal adsorbido en el adsorbente, $C_{\mathrm{e}}$ es la concentración residual del metal en solución, qmax es la adsorción máxima correspondiente a los sitios de saturación, y $b$ es la relación entre la tasas de adsorción/desorción [26].

\section{2) Modelo de Freundlich}

Se basa en una adsorción multicapa en superficies heterogéneas con interacción entre las moléculas adsorbidas y considera una energía de distribución uniforme. La aplicación de este modelo sugiere que la energía decrece exponencialmente con la finalización de los centros de adsorción del adsorbente y se expresa (5): 


$$
q_{e}=K_{f} C_{e}^{1 / n}
$$

Aquí $K_{\mathrm{f}}$ es la constante de Freundlich, $n$ representa la intensidad de adsorción, $q_{\mathrm{e}}$ es la cantidad de metal adsorbido en el equilibrio, y $C_{\mathrm{e}}$ es la concentración residual del metal en solución [27].

\section{RESULTADOS}

\section{A. Caracterización del bioadsorbente}

La pulpa residual del proceso de obtención de almidón a partir de ñame se caracterizó mediante análisis químico bromatológico y FTIR. En la Fig. 1 se muestra el espectro FTIR de la pulpa residual de almidón de ñame antes del proceso de adsorción:

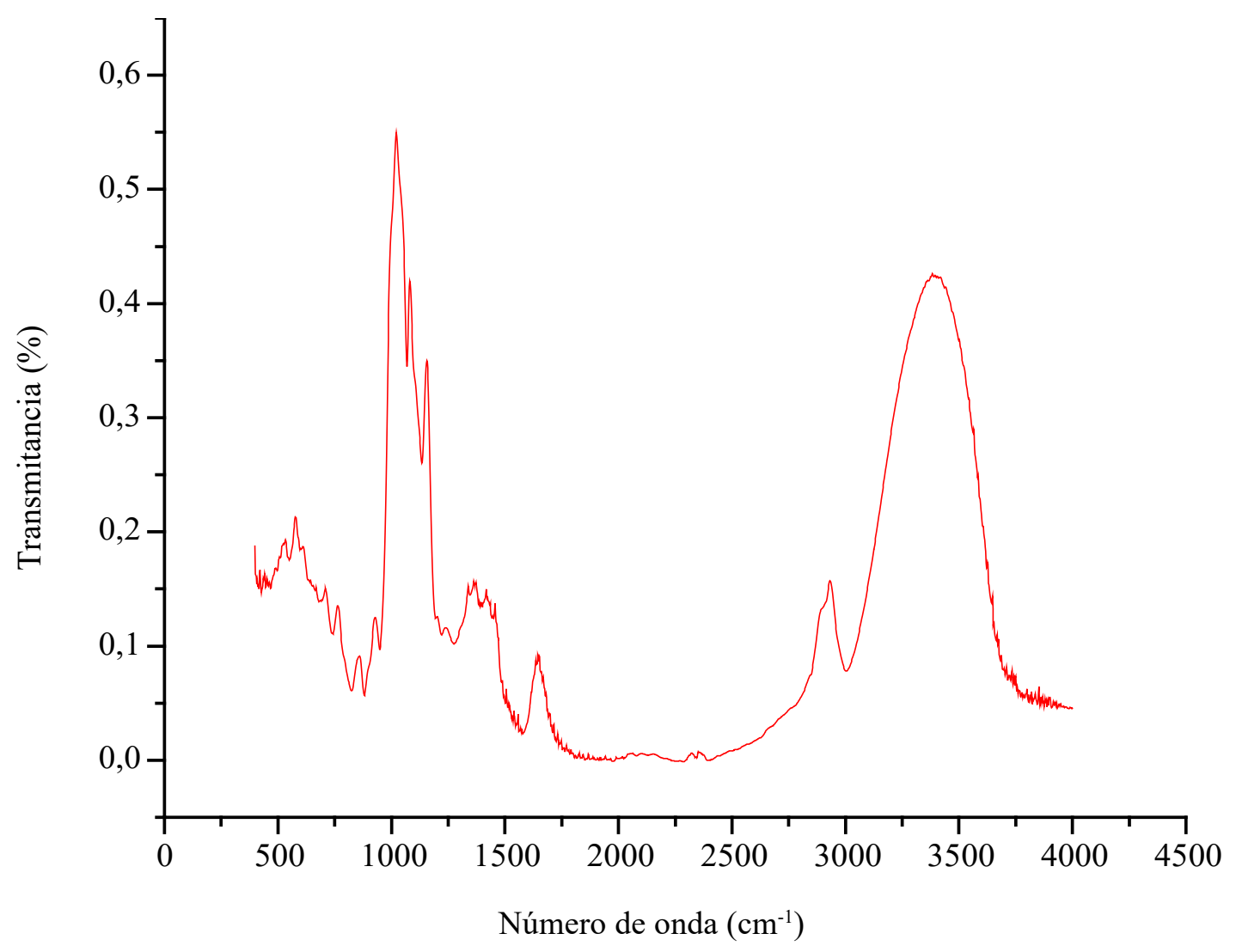

Fig. 1. Espectro FTIR del bioadsorbente Fuente: Autores.

La pulpa residual presentó 19,53\% de lignina, 39,24\% de celulosa y 27,41\% de hemicelulosa [17]. La lignina, celulosa y hemicelulosa son polímeros de cadenas largas ramificadas o lineales que se encuentran presentes en las plantas, y se consideran responsables de la adsorción de los iones metálicos [28], [29]. Los porcentajes de lignina, celulosa y hemicelulosa reportados indican que el proceso de adsorción puede resultar favorable para la remoción de iones de metales pesados, ya que estos compuestos orgánicos presentan diversos grupos funcionales que incrementan la remoción de contaminantes metálicos [30].

En el espectro de la Fig. 1 se muestra inicialmente una banda ancha a $3400 \mathrm{~cm}^{-1}$ que se atribuye a las vibraciones de estiramiento del enlace $\mathrm{OH}$, mientras el pico a $2950 \mathrm{~cm}^{-1}$ y 1350 $\mathrm{cm}^{-1}$ al de la vibración C-H [3]. El pico que aparece a $1650 \mathrm{~cm}^{-1}$ corresponde a la señal intensa característica del estiramiento del grupo carbonilo de los ácidos carboxílicos, al igual que el que aparece a $1400 \mathrm{~cm}^{-1}$ relacionado con la torsión del carboxilo, y a $900 \mathrm{~cm}^{-1}$ de la torsión fuera del plano del dímero del carboxilo [9]. El pico a $1150 \mathrm{~cm}^{-1}$ se relaciona por su parte con el estiramiento del anión COO-, a $1080 \mathrm{~cm}^{-1}$ al estiramiento simétrico y asimétrico del grupo C-O de la vibración de ésteres, y a $1030 \mathrm{~cm}^{-1}$ con el grupo funcional C-O-C [28]. La presencia de grupos hidroxilo, grupo carbonilo, éteres y compuestos aromáticos se deben al alto contenido de celulosa y lignina en el biomaterial, es una evidencia de la estructura lignocelulósica del biomaterial en estudio, y a estos se atribuye la participación en el proceso de adsorción de iones metálicos [31], [32]. 


\section{B. Optimización de variables por la Metodología de Superficie Respuesta (RSM)}

Una vez realizada la experimentación, se aplicó la metodología Superficie Respuesta (RSM) utilizando el software Statgraphics Centurion XVI.II, obteniendo la ecuación de regresión (6) en función de la capacidad de adsorción $q_{\mathrm{e}}$, que ajustó los datos del sistema. La ecuación que ajusta los datos de adsorción de $\mathrm{Cr}(\mathrm{VI})$ es la siguiente:

$$
\begin{aligned}
q_{e}= & -6,01859-0,79674 * \mathrm{~T}+98,0305 \\
& * \mathrm{~A}-0,155557 * \mathrm{C}+0,0104372 * \mathrm{~T}^{2} \\
& -1,26502 * \mathrm{~T} * \mathrm{~A}+0,00537723 * \mathrm{~T} \\
& * \mathrm{C}-3,49734 * \mathrm{~A}^{2}-0,166952 \\
& * \mathrm{~A} * \mathrm{C}+0,000116763 * \mathrm{C}^{2}
\end{aligned}
$$

Donde $\mathrm{T}$ es la temperatura, A la cantidad de adsorbente y C la concentración inicial del metal en solución. Por su parte, los datos de adsorción de Ni(II) son ajustados por la regresión lineal mostrada (7):

$$
\begin{aligned}
q_{e}= & -4,6956-23,224 * A+0,677955 \\
& * T+0,0483815 * C-6,68339 * A^{2} \\
& +0,703582 * A * T+0,014633 * A \\
& * C-0,00371171 * \mathrm{~T}^{2}-0,00293942 \\
& * T * C+0,000213721 * C^{2}
\end{aligned}
$$

Las ecuaciones (6)y (7), se usan para describir la relación entre la capacidad de adsorción $\left(q_{\mathrm{e}}\right)$, dosis de adsorbente, temperatura y concentración inicial de contaminante. Se puede observar la influencia de cada efecto y sus interacciones como valores estandarizados, donde se obtuvo un coeficiente de correlación, R2 de 0,81 para $\mathrm{Cr}$ (VI) y 0,77 para $\mathrm{Ni}(\mathrm{II})$, lo que indicó que el modelo se ajustó en un 80,69 y un $75,64 \%$, respectivamente. Ahora bien, con este modelo se encontró el valor óptimo al cual se realizaron los ensayos cinéticos y de equilibrio de adsorción [33].

\section{Valor óptimo de remoción teórico}

La combinación de los niveles mostrados en la Tabla 2, garantizan la maximización de la capacidad de adsorción de los metales en estudio; esto, debido a la baja cantidad de adsorbente y una alta concentración del metal, obteniendo un valor óptimo teórico de $95 \%$ para $\operatorname{Cr}(\mathrm{VI})$ y un $42 \%$ para Ni(II). Se ha determinado que la concentración en el equilibrio, la biomasa retiene más iones metálicos a una densidad de poros menor que a una mayor. A concentraciones elevadas de biomasa, se presenta un efecto de apantallamiento de los sitios de intercambio por lo denso de la capa celular, es decir, se forma una barrera de electrones de un mismo nivel, que ejerce una fuerza de repulsión sobre electrones de mayor nivel, disminuyendo la probabilidad de encontrar estos electrones en niveles inferiores [34].

TABla 2. VALORES ÓPtimos OPERACIONALES PARA LA OBTENCIÓN DEL PORCENTAJE MÁXIMO DE ADSORCIÓN DE CR(VI) y NI(II)

\begin{tabular}{|l|c|c|l|c|l|l|}
\hline \multirow{2}{*}{ Factor } & \multicolumn{3}{c|}{ Cromo (VI) } & \multicolumn{3}{c|}{ Níquel (II) } \\
\cline { 2 - 7 } & Bajo & \multicolumn{1}{|c|}{ Alto } & Óptimo & Bajo & Alto & Óptimo \\
\hline Temperatura & 29,77 & 80,23 & 76,16 & 29,77 & 70 & 70 \\
\hline Cantidad de adsorbente & 0,14 & 1,22 & 0,14 & 0,135 & 1,22 & 1,19 \\
\hline Concentración inicial & 31,82 & 368,18 & 368,18 & 31,82 & 300 & 31,82 \\
\hline
\end{tabular}

Fuente: Autores.

Estas condiciones óptimas son parte de un estudio básico, que conforman un proceso proyectado a escala industrial en el departamento de Bolívar; en donde, entre otras, se contempla la línea de producción de almidón, y la pulpa, objeto del presente estudio, se presenta como 
una valorización de residuos para su uso como biomaterial adsorbente con diferentes fines, lo cual se muestra como una oportunidad para futuras investigaciones de desarrollo tecnológico e implementación a escala real [35].

\section{Superficie de respuesta}

En las gráficas de superficie de respuesta (Fig. 2) se combinaron los efectos de tres factores principales y el valor de la variable respuesta en todas las combinaciones resultantes a una concentración inicial de 200ppm:
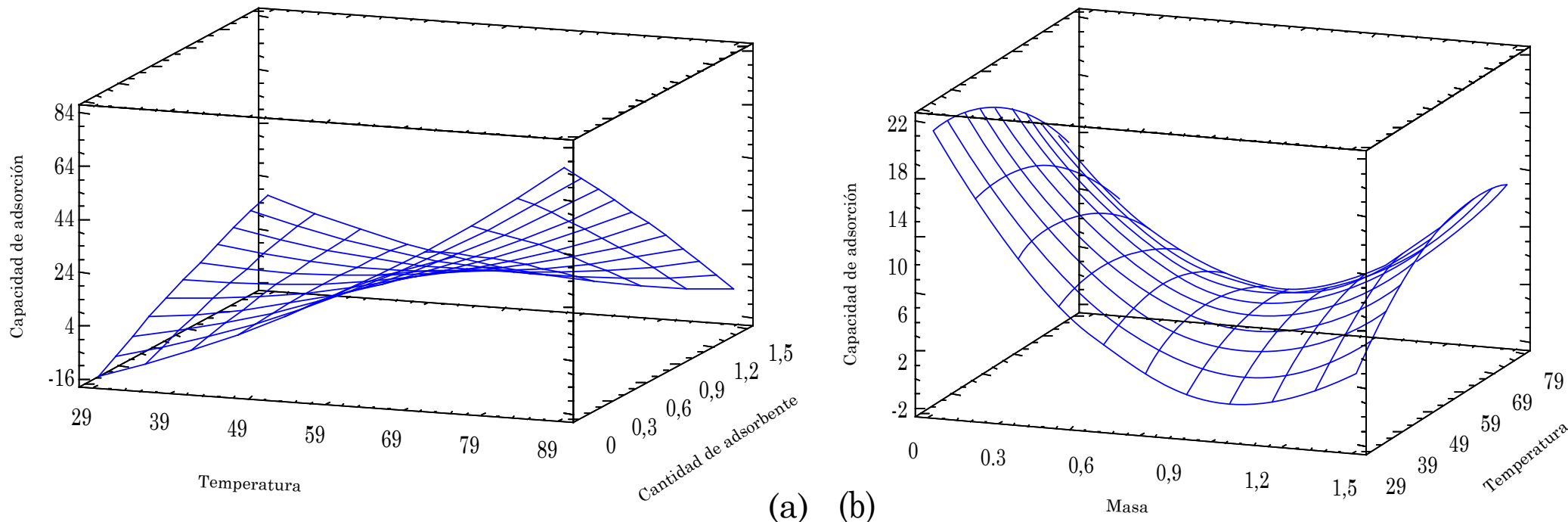

Fig. 2. Superficie de respuesta estimada del valor óptimo de los procesos de adsorción de $\mathrm{Cr}(\mathrm{VI})$ y Ni (II). Fuente: Autores.

De la Fig. 1a se establece que el factor con mayor influencia sobre la capacidad de adsorción de $\mathrm{Cr}(\mathrm{VI})$ es la Temperatura $\left({ }^{\circ} \mathrm{C}\right)$, lo cual está relacionado directamente con la forma de interacción entre la superficie del adsorbente y el ion metálico, lo que puede deberse a la difusión acelerada de los iones metálicos desde la capa externa hacia los poros del adsorbente a medida que aumenta la temperatura [10].

\section{E. Cinética de adsorción}

El estudio cinético se realiza con el fin de conocer el comportamiento del proceso de adsorción en el tiempo. En la Fig. 3a se presenta la curva cinética y los ajustes hechos para la adsorción de $\mathrm{Cr}$ (VI), y de los datos experimentales se observa que el tiempo de equilibrio es de aproximadamente 180 minutos. La Fig. 3b, muestra la curva cinética y ajuste a los modelos para el proceso de remoción de $\mathrm{Ni}(\mathrm{II})$, y se establece una rápida adsorción de los iones metálicos en los primeros 30 min con altas capacidades de adsorción. De la Fig. 3 se puede establecer que los sitios disponibles en el adsorbente comienzan a saturarse y por tanto la capacidad no cambia significativamente a mayores tiempos [4].
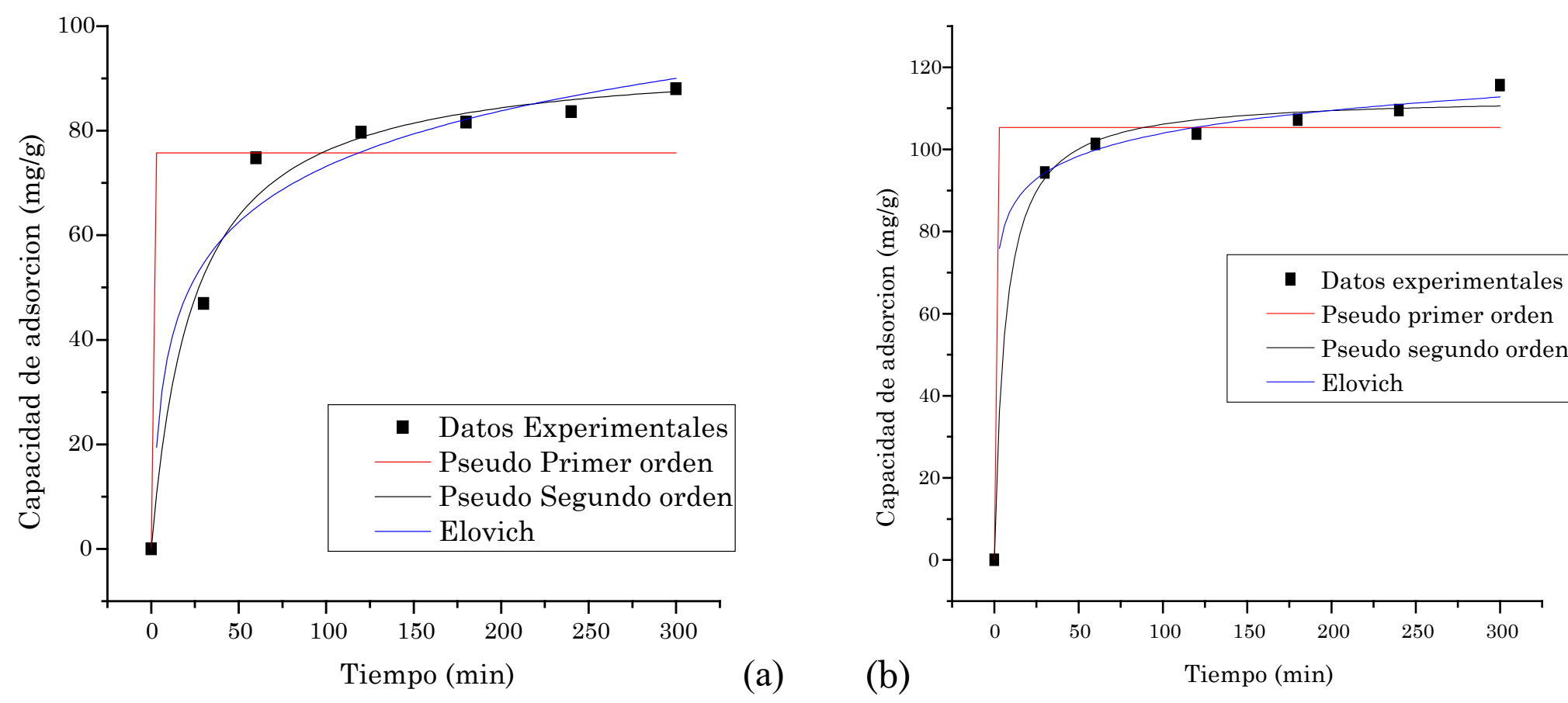

Fig. 3. Ajuste de modelos de la cinética de la adsorción de (a) Cr (VI) y (b) Ni (II). Fuente: Autores. 
Los parámetros de ajuste de los modelos utilizados fueron encontrados por ajuste no lineal, y se muestran en la Tabla 3. Se encontró que el modelo de pseudo segundo orden describe mejor la cinética de adsorción del $\mathrm{Cr}(\mathrm{VI})$, de acuerdo al coeficiente de correlación obtenido; éste modelo representa la quimisorción o adsorción química debida a la formación de enlaces químicos entre adsorbente y adsorbato en una monocapa en la superficie [36]. Del valor de la constante $K_{2}$ se muestra que la tasa de sorción inicial para el cromo es alta, alcanzando también una alta eficiencia de adsorción.

Tabla 3. Parámetros de cinética de la adsorción de Cr (VI).

\begin{tabular}{|l|l|l|l|}
\hline \multirow{2}{*}{ Modelo } & \multirow{2}{*}{ Parámetros } & \multicolumn{2}{c|}{ Metal } \\
\cline { 3 - 4 } & & $\mathrm{Cr}(\mathrm{VI})$ & \multicolumn{1}{c|}{$\mathrm{Ni}(\mathrm{II})$} \\
\hline \multirow{4}{*}{ Pseudo-primer orden } & $q_{\mathrm{e}}\left(\mathrm{mmol}^{-\mathrm{g}}\right)$ & 75,78 & 105,3 \\
\cline { 2 - 4 } & $k_{1}\left(\mathrm{~min}^{-1}\right)$ & $1,70 \mathrm{E} 12$ & $8,195 \mathrm{E} 12$ \\
\cline { 2 - 4 } & $R^{2}$ & 0,7819 & 0,9672 \\
\hline \multirow{4}{*}{ Pseudo-segundo orden } & $q_{\mathrm{e}}\left(\mathrm{mmol}^{\mathrm{g}}\right)$ & 94,60 & 112,9 \\
\cline { 2 - 4 } & $k_{2}\left(\mathrm{~g}^{-1} \mathrm{~min}^{-1}\right)$ & 34903,16 & 223714,75 \\
\cline { 2 - 4 } & $R^{2}$ & 0,9813 & 0,9948 \\
\hline \multirow{3}{*}{ Elovich } & $\alpha$ & 18,002 & 33838,02 \\
\cline { 2 - 4 } & $\beta$ & 0,0651 & 0,1246 \\
\cline { 2 - 4 } & $R^{2}$ & 0,9647 & 0,9979 \\
\hline
\end{tabular}

Fuente: Autores.

Los coeficientes de correlación indican que la cinética de adsorción del níquel bajo las condiciones de estudio se ajustó mejor al modelo de Elovich, con un coeficiente de correlación de 0,99, y el parámetro a, relacionado con la energía de quimiadsorción, de 33838,02 mg/g min, indican que la cinética de adsorción del níquel se ajustó mejor al modelo de Elovich. La ecuación de Elovich es adecuada para describir el comportamiento de adsorción que concuerda con la naturaleza de la adsorción química y supone que los sitios activos del bioadsorbente son heterogéneos y por ello exhiben diferentes energías de activación a lo largo del proceso de adsorción [37].

\section{F. Equilibrio de adsorción}

$\mathrm{El}$ ajuste de los datos experimentales de equilibrio de adsorción de los iones de $\mathrm{Cr}(\mathrm{VI})$ y $\mathrm{Ni}(\mathrm{II})$ se muestran en la Fig. 4. Los parámetros de los modelos de isotermas fueron evaluados por regresión no-lineal y se muestran en la Tabla 4.
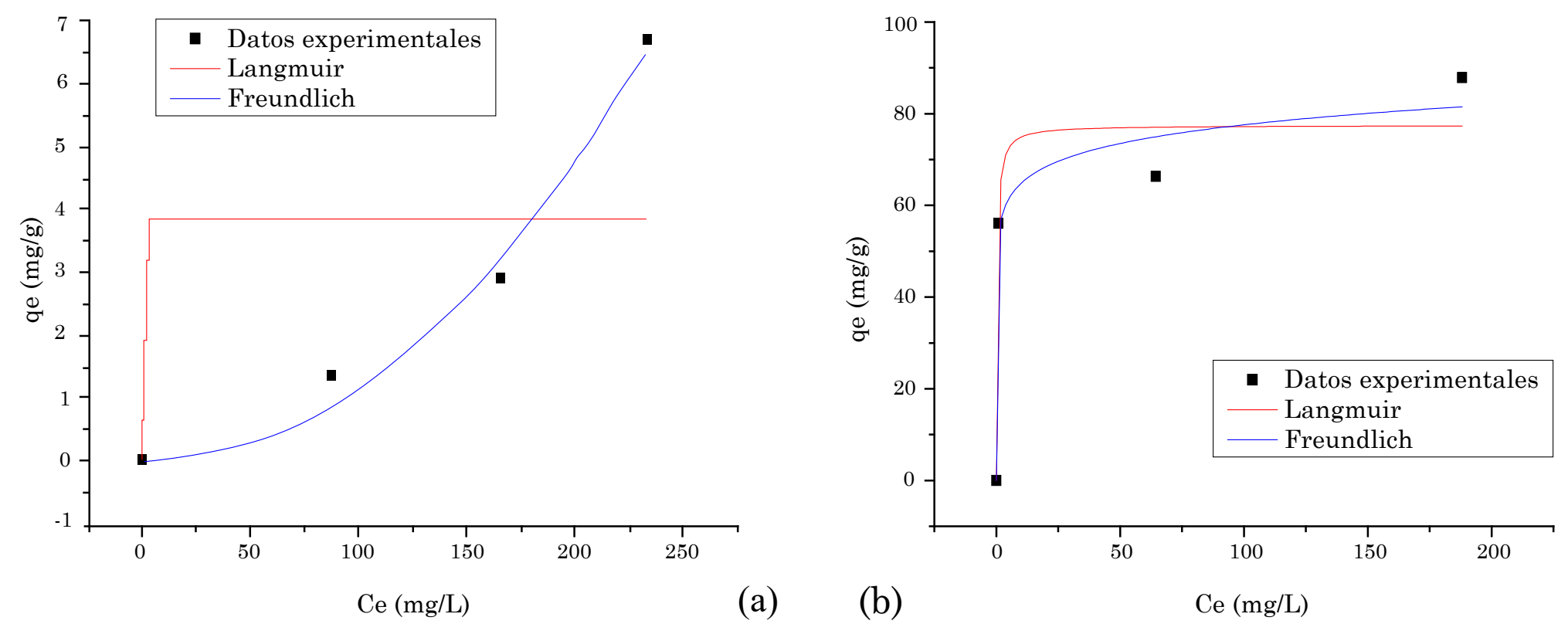

Fig. 4. Ajuste de modelos de las Isotermas de adsorción de (a) Ni (II) y (b) $\mathrm{Cr}$ (VI)

Fuente: Autores.

Los valores de los parámetros reportados en la Tabla 4, indican que la máxima capacidad de adsorción de $\mathrm{Cr}(\mathrm{VI})$ en la monocapa es de 77,46 mg/g, de acuerdo con el modelo de Langmuir. 
Se observa en la Fig. 4 que el modelo que mejor ajusta el equilibrio de adsorción de Cromo y Níquel es el de Freundlich, con una constante n de 12,82 y 0,49, lo cual indica que los enlaces químicos formados entre el Cr(VI) y adsorbente son fuertes, y que el proceso de adsorción es favorable [38].

Tabla 4. Parámetros de Freundlich y Langmuir para adsorción de Ni (II)

\begin{tabular}{|c|c|c|c|}
\hline \multirow{2}{*}{ Modelo } & \multirow{2}{*}{ Parámetro } & \multicolumn{2}{|c|}{ Metal } \\
\hline & & $\mathrm{Cr}(\mathrm{VI})$ & $\mathrm{Ni}(\mathrm{II})$ \\
\hline \multirow{3}{*}{ Langmuir } & $q_{\max }(\mathrm{mg} / \mathrm{g})$ & 77,461 & $3,86 \times 108$ \\
\hline & $b(\mathrm{~L} / \mathrm{mg})$ & 2,915 & $3,86 \times 1038$ \\
\hline & $R^{2}$ & 0,9197 & 0,0811 \\
\hline \multirow{3}{*}{ Freundlich } & $K_{\mathrm{f}}$ & 54,17 & $8,73 \times 10-5$ \\
\hline & $n$ & 12,82 & 0,49 \\
\hline & $R^{2}$ & 0,9571 & 0,9773 \\
\hline
\end{tabular}

Fuente: Autores.

Por su parte, la intensidad de adsorción para el Ni(II), dada por el parámetro de Freundlich (n) es baja, 0.49, lo que indica que la unión de los iones de Ni (II) en la superficie del adsorbente es débil [39]. Aunque este parámetro es bajo, el modelo que mejor se ajusta a los datos, es el de Freundlich, ya que no establece un valor límite de la cantidad adsorbida, además de tener en cuenta aquellos comportamientos no lineales ente la cantidad adsorbida y la concentración de la fase fluida, mientras que el modelo de Langmuir dispone de un numero finito de sitios de adsorción. Asimismo, el coeficientes de correlación sugiere que el proceso de adsorción de $\mathrm{Ni}(\mathrm{II})$ puede darse por multicapa y no por monocapa.

La intensidad de adsorción, dada por el parámetro de Freundlich (n) es alta en comparación con otros trabajos usando materiales adsorbentes de origen vegetal: 1,55-5,74 para el Cr(VI) [49], [50]; y 0.592-6.5789 para el Ni(II) [1], [12]-[13], [40]-[41]. El parámetro de Freundlich $(n)$ confirmó que el proceso puede ser clasificado como favorable para la adsorción [51].

\section{G. Estudio termodinámico}

Los parámetros termodinámicos del proceso de adsorción de Cr (VI) y Ni (II) se calcularon con la finalidad de establecer la espontaneidad, favorabilidad y la influencia de la temperatura en el proceso; los parámetros determinados fueron los valores de entalpia de adsorción $\left(\Delta \mathrm{H}^{\circ}\right)$, entropía de adsorción $\left(\Delta \mathrm{S}^{\circ}\right)$ y energía de Gibbs $\left(\Delta \mathrm{G}^{\mathrm{o}}\right)$, mediante el método gráfico de Van't Hoff.

De acuerdo a los parámetros calculados en el proceso de adsorción de Cr (VI), se establece que el proceso de adsorción se favorece con el aumento de la temperatura, encontrando un valor de $\Delta \mathrm{H}$ positivo, lo que indica que el proceso es endotérmico [42]. Los valores positivos de $\Delta \mathrm{G}$ indican la no espontaneidad del proceso de adsorción para esta biomasa, ya que el sistema no es capaz de evolucionar por sí solo. Es necesario aportar energía al sistema para que el proceso sea favorable, lo que justificaría porque la adsorción aumenta ligeramente con la temperatura. La magnitud del valor de $\Delta \mathrm{H}$ cercano a $40 \mathrm{KJ} / \mathrm{mol}$ sugiere la coexistencia de adsorción física y química. Mientras tanto el valor obtenido para $\Delta \mathrm{S}$, signo y magnitud baja, indica que este proceso es reversible [43]-[45].

Tabla 5. Parámetros termodinámicos para la adsorción de Cr (VI) y Ni (II)

\begin{tabular}{|l|l|c|c|c|c|c|}
\hline \multirow{2}{*}{$\mathrm{T}(\mathrm{K})$} & \multicolumn{3}{|c|}{$\mathrm{Cr}(\mathrm{VI})$} & \multicolumn{3}{c|}{$\mathrm{Ni}(\mathrm{II})$} \\
\cline { 2 - 7 } & $\Delta \mathrm{G}(\mathrm{kJ} / \mathrm{mol})$ & $\Delta \mathrm{H}(\mathrm{kJ} / \mathrm{mol})$ & $\Delta \mathrm{S}(\mathrm{kJ} / \mathrm{mol} . \mathrm{K})$ & $\Delta \mathrm{G}(\mathrm{kJ} / \mathrm{mol})$ & $\Delta \mathrm{H}(\mathrm{kJ} / \mathrm{mol})$ & $\Delta \mathrm{S}(\mathrm{kJ} / \mathrm{mol} . \mathrm{K})$ \\
\hline 30 & 16,33 & 39,73 & 0,0772 & 17,34 & $-0,2082$ & $-0,0579$ \\
\hline 55 & 14,40 & - & - & 18,79 & - & - \\
\hline 80 & 12,47 & - & - & 0 & - & - \\
\hline
\end{tabular}

Fuente: Autores. 
Según los parámetros termodinámicos mostrados en la Tabla 5 para la remoción de Ni (II), los valores positivos obtenidos a 30 y $55^{\circ} \mathrm{C}$ para la energía libre de Gibbs sugieren que el proceso de adsorción es no espontaneo a las temperaturas evaluadas [46]. Con relación a la entalpía de adsorción cuyo valor es negativo $(-0,2082 \mathrm{~kJ} / \mathrm{mol})$ implica que el proceso de adsorción es exotérmico; por esto, se puede decir, que el proceso de remoción de $\mathrm{Ni}$ (II) se debe a la fisisorción y que las interacciones sorbato-sorente son principalmente electrostática. En cuanto a la entropía se obtuvo un valor negativo de $-0,0579 \mathrm{~kJ} / \mathrm{mol}$, lo que indicó que las moléculas de níquel están organizadas más regularmente en la superficie del adsorbente, es decir, hay una disminución de la aleatoriedad en la interfase sólidoliquido [47], [48].

\section{Conclusiones}

Del análisis FTIR del adsorbente se establece que es un material heterogéneo con presencia de grupos funcionales hidroxilo, carboxilo, carbonilo, hidrocarburos insaturados, entre otros, que son propios de los materiales ricos en lignina y celulosa, y que actúan como centros activos intervinientes en el proceso de remoción. Se encontró que la condición óptima para la adsorción de $\mathrm{Cr}(\mathrm{VI})$ una temperatura de $76,2^{\circ} \mathrm{C}$, cantidad de adsorbente de 0,135 g y concentración inicial 368,2 ppm; por su parte para el $\mathrm{Ni}$ (II) las condiciones óptimas operacionales fueron $70^{\circ} \mathrm{C}$, con dosis de adsorbente de $1.19 \mathrm{~g}$ y $38.1 \mathrm{ppm}$. Se logró una máxima capacidad de remoción de $66,35 \mathrm{mg} / \mathrm{g}$ para el $\mathrm{Cr}(\mathrm{VI})$ y de $17,67 \mathrm{mg} / \mathrm{g}$ para el $\mathrm{Ni}(\mathrm{II})$. Los datos experimentales de $\mathrm{Cr}(\mathrm{VI})$ se ajustaron al modelo de pseudo-segundo orden y los de $\mathrm{Ni}(\mathrm{II})$ al de Elovich, lo que indica la formación de enlaces químicos entre adsorbente y adsorbato. Por su parte, el proceso de adsorción de $\mathrm{Cr}(\mathrm{VI})$ y $\mathrm{Ni}(\mathrm{II})$ se ajustó al modelo de Freundlich con un coeficiente de intensidad $n$ de 12,82 y 0,49 , respectivamente; lo que indica que la adsorción del Cromo tiene lugar en superficies heterogéneas, mientras que los iones de Níquel se adhieren débilmente a la superficie del adsorbente. Del estudio termodinámico se establece que el proceso de adsorción de $\mathrm{Cr}(\mathrm{VI})$ es endotérmico, no espontáneo no favorable y reversible; de otra parte, se establece que el proceso de remoción de Ni(II) es es no espontaneo, exotérmico y controlado por fisisorción. Éste estudio se presenta como una alternativa para la disposición final de residuos del proceso de obtención de almidón a partir de ñame, y su uso como biomaterial representa una solución para mitigar la contaminación por Cromo(VI).

\section{Financiamiento}

Artículo de investigación científica derivado del proyecto de Fortalecimiento de Grupo "Aprovechamiento de residuos agrícolas y agroindustriales del departamento de Bolívar para la bioadsorción de cromo y níquel, contenido en aguas contaminadas por la actividad de las microempresas de cromado y niquelado de la ciudad de Cartagena," financiado por la Universidad de Cartagena mediante Convocatoria de Vicerrectoría de Investigaciones 2017.

\section{REFERENCIAS}

[1] A. Haq, M. Saeed, S. Anjum, T. H. Bokhari, M. Usman and S. Tubbsum, "Evaluation of Sorption Mechanism of $\mathrm{Pb}$ ( II ) and Ni ( II ) onto Pea ( Pisum sativum ) Peels," J. Oleo Sci., vol. 66, no. 7, pp. 735-743, Jun. 2017. https://doi.org/10.5650/jos.ess17020

[2] S. Ratan, I. Singh, J. Sarkar and N. Rm, "The Removal of Nickel from Waste Water by Modified Coconut Coir Pith," Chem. Sci. J., vol. 7, no. 3, pp. 1-6, Jun. 2016. Available from https://www.hilarispublisher.com/open-access/the-removal-of-nickel-from-waste-water-by-modified-coconut-coirpith-2150-3494-1000136.pdf

[3] E. Ben, B. Rzig, R. Chakroun, H. Nouagui and B. Hamrouni, "Application of response surface methodology for chromium removal by adsorption on low-cost biosorbent," Chemom. Intell. Lab. Syst., vol. 189, pp. 18-26, Jun. 2019. https://doi.org/10.1016/j.chemolab.2019.03.014

[4] A. Villabona-Ortíz, C. Tejada-Tovar and R. Ortega-Toro, "Modelling of the adsorption kinetics of Chromium (VI) using waste biomaterials," Rev. Mex. Ing. Química, vol. 19, no. 1, pp. 401-408, Jan. 2020. https://doi.org/10.24275/rmiq/IA650

[5] A. Kumar et al., "Remediation techniques applied for aqueous system contaminated by toxic Chromium and Nickel ion," Geol. Ecol. Landscapes, vol. 1, no. 2, pp. 143-153, Jun. 2017. https://doi.org/10.1080/24 749508.2017.1332860 
[6] I. Enniya, L. Rghioui and A. Jourani, "Adsorption of hexavalent chromium in aqueous solution on activated carbon prepared from apple peels," Sustain. Chem. Pharm., vol. 7, no. 1, pp. 9-16, Mar. 2018. https://doi.org/10.1016/j.scp.2017.11.003

[7] A. Witek-Krowiak, K. Chojnacka, D. Podstawczyk, A. Dawiec and K. Pokomeda, "Application of response surface methodology and artificial neural network methods in modelling and optimization of biosorption process," Bioresour. Technol., vol. 160, no. 1, pp. 150-160, May. 2014. https://doi.org/10.1016/j. biortech.2014.01.021

[8] M. Manjuladevi, R. Anitha and S. Manonmani, "Kinetic study on adsorption of Cr (VI), Ni (II), Cd (II) and $\mathrm{Pb}$ (II) ions from aqueous solutions using activated carbon prepared from Cucumis melo peel," Appl. Water Sci., vol. 8, no. 1, pp. 1-8, Feb. 2018. https://doi.org/10.1007/s13201-018-0674-1

[9] S. Afroze and T. K. Sen, "A Review on Heavy Metal Ions and Dye Adsorption from Water by Agricultural Solid Waste Adsorbents," Water, Air Soil Pollut., vol. 229, no. 7, p. 225, Jun. 2018. https://doi. org/10.1007/s11270-018-3869-z

[10] Y. Wu, Y. Fan, M. Zhang, Z. Ming, S. Yang and A. Arkin, "Functionalized agricultural biomass as a low-cost adsorbent: Utilization of rice straw incorporated with amine groups for the adsorption of $\mathrm{Cr}$ ( VI ) and Ni ( II ) from single and binary systems," Biochem. Eng. J., vol. 105, pp. 27-35, Jan. 2016. https://doi.org/10.1016/j.bej.2015.08.017

[11] A. Razafsha, P. Ziarati and M. Moslehishad, "Removal of Heavy Metals from Oryza sativa Rice by Sour Lemon Peel as Bio-sorbent," Biomed. Pharmacol. J., vol. 9, no. 2, pp. 543-553, Aug. 2016. https://doi. org/10.13005/bpj/971

[12] Y. F. Lam, L. Yee, S. J. Chua, S. S. Lim and S. Gan, "Ecotoxicology and Environmental Safety Insights into the equilibrium , kinetic and thermodynamics of nickel removal by environmental friendly Lansium domesticum peel biosorbent," Ecotoxicol. Environ. Saf., vol. 127, no. 1, pp. 61-70, May. 2016. https:// doi.org/10.1016/j.ecoenv.2016.01.003

[13] Y. Yi, J. Lv, Y. Liu and G. Wu, "Synthesis and application of modified Litchi peel for removal of hexavalent chromium from aqueous solutions," J. Mol. Liq., vol. 225, pp. 28-33, Jan. 2017. https://doi.org/10.1016/j. molliq.2016.10.140

[14] S. Kuppusamy, P. Thavamani, Y. B. Lee and R. Naidu, "Oak (Quercus robur ) Acorn Peel as a Low-Cost Adsorbent for Hexavalent Chromium Removal from Aquatic Ecosystems and Industrial Effluents," Water, Air, Soil Pollut., vol. 227, no. 2, pp. 1-11, Jan. 2016. https://doi.org/10.1007/s11270-016-2760-z

[15] C. Tejada-Tovar, J. Gallo-Mercado, J. Moscote, A. Villabona-Ortíz and D. Acevedo-Correra, "Adsorción competitiva de plomo y niquel sobre cáscara de ñame y bagazo de palma en sistema continuo," BSAA, vol. 16, no. 1, pp. 52-61, Ene. 2018. Disponible en https://revistas.unicauca.edu.co/index.php/biotecnologia/article/view/1145

[16] R. E. Tejada-Tovar, L. P. Tejeda-Benítez, C. Tejada-Tovar, A. Villabona-Ortíz and C. Granados-Conde, "Aprovechamiento de ñame espino post-cosecha (Dioscorea rotundata P.) en la extracción de ácido láctico in the extraction of lactic acid," Prospectiva, vol. 16, no. 1, pp. 76-82, Dic. 2018. https://doi.org/10.15665/ rp.v16i1.1429

[17] B. C. Maniglia and D. R. Tapia-Blácido, "Food Hydrocolloids Isolation and characterization of starch from babassu mesocarp," vol. 55, pp. 47-55, Apr. 2016. https://doi.org/10.1016/j.foodhyd.2015.11.001

[18] C. Tejada-Tovar, A. Villabona-Ortíz, Á. D. González-Delgado, C. Granados-Conde and M. Jiménez-Villadiego, "Kinetics of Mercury and Nickel adsorption using chemically pretrated cocoa (Theobroma cacao) husk," Trans. ASABE, vol. 62, no. 2, pp. 461-466, Jan. 2019. https://doi.org/10.13031/trans.13133

[19] M. Iqbal, N. Iqbal, I. Ahmad, N. Ahmad and M. Zahid, "Response surface methodology application in optimization of cadmium adsorption by shoe waste: A good option of waste mitigation by waste," Ecol. Eng., vol. 88, no. 1, pp. 265-275, Mar. 2016. https://doi.org/10.1016/j.ecoleng.2015.12.041

[20] C. Tejada-Tovar, Á. Villabona-Ortiz and M. Jiménez-Villadiego, "Remoción de cromo hexavalente sobre residuos de cacao pretratados químicamente," Rev. U.D.C.A Act. \& Div. Cient., vol. 10, no. 1, pp. 139-147, Jun. 2017. https://doi.org/10.31910/rudca.v20.n1.2017.71

[21] C. T. Tovar, Á. V. Ortiz and M. J. Villadiego, "Remoción de cromo hexavalente sobre residuos de cacao pretratados químicamente," Rev. U.D.C.A Act. \& Div. Cient., vol. 20, no. 1, pp. 139-147, Jun. 2017. https://doi.org/10.31910/rudca.v20.n1.2017.71

[22] C. Tien and B. V. Ramarao, "On the significance and utility of the Lagergren model and the pseudo second-order model of batch adsorption,” Sep. Sci. Technol., vol. 52, no. 6, pp. 975-986, Dec. 2016. https:// doi.org/10.1080/01496395.2016.1274327

[23] Y. S. Ho and G. Mckay, "Pseudo-second order model for sorption processes," Process Biochem., vol. 34, no. 5, pp. 451-465, Jul. 1999. https://doi.org/10.1016/S0032-9592(98)00112-5

[24] W. Marimón-Bolívar, L. Tejeda-Benítez and A. P. Herrera, "Removal of mercury (II) from water using magnetic nanoparticles coated with amino organic ligands and yam peel biomass," Environ. Nanotechnol. Monit. Manage, vol. 10, pp. 486-493, Dec. 2018. https://doi.org/10.1016/j.enmm.2018.10.001

[25] C. Aharoni and F. C. Tompkins, "Kinetics of Adsorption and Desorption and the Elovich Equation," Adv. Catal., vol. 21, pp. 1-49, 1970. https://doi.org/10.1016/S0360-0564(08)60563-5

[26] I. Langmuir, "The constitution and fundamental properties of solids and liquids. Part I. Solids.," J. Am. Chem. Soc., vol. 38, no. 11, pp. 2221-2295, Nov. 1916. https://doi.org/10.1021/ja02268a002

[27] J. Skopp, "Derivation of the Freundlich Adsorption Isotherm from Kinetics," J. Chem. Educ., vol. 86, no. 11, pp. 1341-1343, Nov. 2009. https://doi.org/10.1021/ed086p1341 
[28] D. L. Gómez, J. P. Rodríguez, J. A. Esteban and J. F. Betancur, "Coffee Pulp: A Sustainable Alternative Removal of Cr (VI) in Wastewaters. Processes," Processes, vol. 7, no. 7, p. 1-12, Jul. 2019. https://doi. org/10.3390/pr7070403

[29] S. Kulbir, W. S. Abdullahi and R. Chhotu, "Removal of Heavy Metals by Adsorption using Agricultural based Residue: A Review," Res. J. Chem. Environ., vol. 22, no. 5, pp. 65-74, May. 2018. Available from https://worldresearchersassociations.com/Archives/RJCE/Vol(22)2018/May2018.aspx

[30] A. Rodriguez, D. Lemos, Y. T. Trujillo, J. G. Amaya and L. D. Ramos, "Effectiveness of Biochar Obtained from Corncob for Immobilization of Lead in Contaminated Soil," J. Heal. Polution, vol. 9, no. 23, pp. 1-10, Sept. 2019. https://doi.org/10.5696/2156-9614-9.23.190907

[31] X. H. Vu et al., "Adsorption of Chromium (VI) onto Freshwater Snail Shell-Derived Biosorbent from Aqueous Solutions: Equilibrium, Kinetics , and Thermodynamics," J. Chem., vol. 2019, no. 3, pp. 1-11, Sept. 2019. https://doi.org/10.1155/2019/3038103

[32] S. M. Batagarawa and A. K. Ajibola, "Comparative evaluation for the adsorption of toxic heavy metals on to millet, corn and rice husks as adsorbents," J. Anal. Pharm. Res., vol. 8, no. 3, pp. 119-125, May. 2019. https://doi.org/10.15406/japlr.2019.08.00325

[33] S. M. Anisuzzaman, A. Bono, D. Krishnaiah and Y. Z. Tan, "A study on dynamic simulation of phenol adsorption in activated carbon packed bed column," J. King Saud Univ.-Eng. Sci., vol. 28, no. 1, pp. 47-55, Jan. 2016. https://doi.org/10.1016/j.jksues.2014.01.001

[34] Ch. Zhu et al., "Nitrogen-doped chitosan-Fe ( III ) composite as a dual-functional material for synergistically enhanced co-removal of $\mathrm{Cu}$ ( II ) and Cr ( VI ) based on adsorption and redox," Chem. Eng. J., vol. 306, no. C, pp. 579-587, Dec. 2016. https://doi.org/10.1016/j.cej.2016.07.096

[35] P. Ray, M. A. Sabri, T. H. Ibrahim, M. I. Khamis and F. H. Jumean, "Design and optimization of a batch sequential contactor for the removal of chromium ( VI) from industrial wastewater using sheep wool as a low-cost adsorbent," Desalin. Water Treat., vol. 113, pp. 109-113, May. 2018. https://doi.org/10.5004/ dwt.2018.22365

[36] K. M. Doke and E. M. Khan, "Equilibrium, kinetic and diffusion mechanism of $\mathrm{Cr}$ ( VI ) adsorption onto activated carbon derived from wood apple shell," Arab. J. Chem., vol. 10, no. Supplement 1, pp. S252S260, Feb. 2017. https://doi.org/10.1016/j.arabjc.2012.07.031

[37] X. Hu, Y. Xue, L. Liu, Y. Zeng and L. Long, "Preparation and characterization of Na2S-modified biochar for nickel removal," Environ. Sci. Pollut. Res., vol. 25, no. 10, pp. 9887-9895, Jan. 2018. https://doi. org/10.1007/s11356-018-1298-6

[38] A. Ali, K. Saeed and F. Mabood, "Removal of chromium ( VI ) from aqueous medium using chemically modified banana peels as efficient low-cost adsorbent," Alexandria Eng. J., vol. 55, no. 3, pp. 2933-2942, Sep. 2016. https://doi.org/10.1016/j.aej.2016.05.011

[39] Z. N. Garba, N. I. Ugbaga and A. K. Abdullahi, "Evaluation of optimum adsorption conditions for Ni ( II ) and Cd ( II ) removal from aqueous solution by modified plantain peels ( MPP )," Beni-Suef Univ. J. Basic Appl. Sci., vol. 5, no. 2, pp. 170-179, Jun. 2016. https://doi.org/10.1016/j.bjbas.2016.03.001

[40] Z. Shen, Y. Zhang, O. McMillan, F. Jin and A. Al-Tabbaa, "Characteristics and mechanisms of nickel adsorption on biochars produced from wheat straw pellets and rice husk," Environ. Sci. Pollut. Res., vol. 24, no. 14, pp. 12809-12819, Mar. 2017. https://doi.org/10.1007/s11356-017-8847-2

[41] R. Sudha, K. Srinivasan and P. Premkumar, "Ecotoxicology and Environmental Safety Removal of nickel (II) from aqueous solution using Citrus Limettioides peel and seed carbon," Ecotoxicol. Environ. Saf., vol. 117, pp. 115-123, Jul. 2015. https://doi.org/10.1016/j.ecoenv.2015.03.025

[42] C. Lin, W. Luo, T. Luo, Q. Zhou, H. Li and L. Jing, "A study on adsorption of Cr (VI) by modified rice straw: Characteristics, performances and mechanism," J. Clean. Prod., vol. 196, pp. 626-634, Sep. 2018. https://doi.org/10.1016/j.jclepro.2018.05.279

[43] E. Rodrigues, O. Almeida, H. Brasil, D. Moraes and M. A. L. dos Reis, "Applied Clay Science Adsorption of chromium ( VI ) on hydrotalcite-hydroxyapatite material doped with carbon nanotubes : Equilibrium , kinetic and thermodynamic study," Appl. Clay Sci., vol. 172, pp. 57-64, May. 2019. https://doi. org/10.1016/j.clay.2019.02.018

[44] V. Manirethan, K. Raval, R. Rajan, H. Thaira and R. M. Balakrishnan, "Kinetic and thermodynamic studies on the adsorption of heavy metals from aqueous solution by melanin nanopigment obtained from marine source : Pseudomonas stutzeri," J. Environ. Manage., vol. 214, pp. 315-324, May. 2018. https:// doi.org/10.1016/j.jenvman.2018.02.084

[45] S. Kar and S. Equeenuddin, "Adsorption of Hexavalent Chromium using Natural Goethite : Isotherm , Thermodynamic and Kinetic Study," J. Geol. Soc. India, vol. 93, no. 3, pp. 285-292, Mar. 2019. https:// doi.org/10.1007/s12594-019-1175-z

[46] S. Alam et al., "Thermodynamic Analysis of Nickel (II) and Zinc (II) Adsorption to Biochar," Environ. Sci., vol. 52, no. 11, pp. 6246-6255, May. 2018. https://doi.org/10.1021/acs.est.7b06261

[47] X. Fan, J. Xia and J. Long, "The Potential of Nonliving Sargassum hemiphyllum as a Biosorbent for Nickel (II) Removal - Isotherm , Kinetics, and Thermodynamics Analysis," Environ. Prog. Sustain. Energy, vol. 38, no. S1, pp. S250-S259, Oct. 2018. https://doi.org/10.1002/ep.12997

[48] J. Long, X. Huang, X. Fan, Y. Peng and J. Xia, "Effective adsorption of nickel (II) with Ulva lactuca dried biomass: isotherms, kinetics and mechanisms," Water Sci. Technol., vol. 78, no. 1, pp. 1-9, Jun. 2018. https://doi.org/10.2166/wst.2018.253 
[49] S. Rangabhashiyam and N. Selvaraju, "Adsorptive remediation of hexavalent chromium from synthetic wastewater by a natural and $\mathrm{ZnCl} 2$ activated Sterculia guttata shell," J. Mol. Liq., vol. 207, pp. 39-49, Jul. 2015. https://doi.org/10.1016/j.molliq.2015.03.018

[50] M. Mishra, J. Huang and M. K. Balasubramanian, "The yeast actin cytoskeleton," FEMS Microbiol Rev., vol. 38, no. 2, pp. 213-217, Feb. 2014. https://doi.org/10.1111/1574-6976.12064

[51] S. R. Irani et al., "Faciobrachial dystonic seizures precede Lgi1 antibody limbic encephalitis," Ann Neurol., vol. 69, no. 5, pp. 892-900, May. 2011. https://doi.org/1002/ana.22307

Angel Villabona Ortíz es profesor en el Departamento de Ingeniería Química y del programa de Ingeniería Química en la Universidad de Cartagena (Colombia) y parte del Process Design and Biomass Utilization Research Group (IDAB). Magister en Ingeniería Ambiental por la Universidad de Cartagena con investigaciones centradas en el tratamiento del agua, adsorción y medio ambiente. https://orcid.org/0000-0001-8488-1076

Candelaria Tejada Tovar es profesor en el Departamento de Ingeniería Química y del programa de Ingeniería Química en la Universidad de Cartagena (Colombia). Parte del Process Design and Biomass Utilization Research Group (IDAB). Magister en Ingeniería Ambiental por la Universidad de Cartagena con investigaciones centradas en tratamiento del agua, adsorción y medio ambiente. https://orcid.org/0000-0002-2323-1544

Paula Ramírez Vásquez es Ingeniera Química de la Universidad de Cartagena (Colombia). Sus investigaciones se centran en tratamiento del agua, adsorción y medio ambiente. https:// orcid.org/0000-0002-0145-232X 